\title{
Research on the 4G Mobile Communication Systems Link Scheme Based on MIMO Systems
}

\author{
Chen Wen-ting ${ }^{1, \text { a }}$ \\ ${ }^{1}$ Chongqing College Of Electronic Engineering, Chongqing 401331, China \\ a37464351@qq.com
}

Keywords: 4G Mobile Communication Systems, Link Scheme, MIMO Systems

\begin{abstract}
With the rapid development of mobile communication technology, 3G mobile communication technology boom has not yet subsided, we have ushered in a more secure, more efficient, faster 4G mobile communications technology. The rapid development of 4G mobile communication systems and channel coding schemes are inseparable, this article on the link scheme 4G mobile communication system design analysis and research, and thus can reliably for 4G mobile communication system, channel coding and the effectiveness of the communication transmission sex, higher solution play a role in promoting the development and improvement of the future $4 \mathrm{G}$ mobile communication system.
\end{abstract}

\section{Introduction}

In the present life, the mobile communications business is in rapid development, the number of users is constantly increasing, and information network multimedia services is endless, so there will be new multimedia access capability but also large capacity mobile communication system, which makes the generation 3G. But until now, many people have found it insufficient, and 4G mobile communications also capable of rendering high-speed data transmission, to achieve voice and multimedia services. This makes life richer side, but it is also facing further challenges, such as multipath fading and bandwidth utilization, so OFDM technique will be able to use the channel in such a manner decomposed into a plurality of orthogonal sub-channel, thereby this solves the frequency selective multipath fading channel conversion to flat fading channel, reducing the effects of multipath fading. Features MIMO technology that allows space to produce a plurality of independent and is peer-channel system, so that you can work together to transmit data at the same time effectively improve the spectrum utilization. So, for now, to be combined with OFDM and MIMO has become the main trend of the new generation of mobile communications.

\section{The Concepts of 4G Mobile Communication System}

Concept of fourth generation mobile communication technology (4G) can be referred to as wideband (Broad-band) access and distribution network, with more than $2 \mathrm{Mb} / \mathrm{s}$ asymmetric data transmission capability for full speed mobile users to provide $150 \mathrm{Mb} / \mathrm{s}$ high-quality video services and high-quality transmission for the first time three-dimensional image. It includes a wide band fixed wireless access, wideband wireless local area network (WLAN), wide band mobile broadcast network systems and interoperability. In different fixed wireless platforms and across different frequency bands networks, 4G wireless services can be provided, and anywhere broadband access to the Internet (including satellite communications layer communication peace flow), providing location other than the timing of information and communication, data acquisition, remote control and other integrated functions.

Currently, industry and academia generally considered: 4G is basically based on IP, with more than $2 \mathrm{Mb} / \mathrm{s}$ asymmetric data transmission capability in a mobile environment to achieve a rate of $100 \mathrm{Mb} / \mathrm{s}$, in a static environment, the rate reached $1 \mathrm{~GB} / \mathrm{s}$ or more, and be able to support the next generation network applications (such as mobile high-definition television), and can easily switch between fixed and mobile technology.

$4 \mathrm{G}$ will be using a single worldwide cellular core network to replace the $3 \mathrm{G}$ cellular network in 
dense, its all-digital all-IP technology. This is from the inner edge of the network and the intelligent network to the entire network of intelligent development. Core network to support different access methods, such as IEEE802.11a, WCDMA, Bluetooth and so on, and each user can have a unique device identification number, interoperability between heterogeneous systems through a hierarchical structure. This configuration allows multiple services can be transparently connected to the IP core network, has good versatility and scalability.

\section{The Key Technology of 4G Mobile Communication System}

Orthogonal Frequency Division Multiplexing Technology (OFDM). Fourth generation mobile communication system is mainly based on orthogonal frequency division multiplexing (OFDM) as the core technology [4]. The main idea of OFDM technology is given in the frequency domain channel into many orthogonal sub-channel narrow, using a subcarrier is modulated on each sub-channel, parallel and each sub-carrier transmission and it can greatly eliminate the interference between the signal waveform. In an OFDM system due to the various sub-carriers orthogonal to each other, so their spectrum is overlapping, it will not only reduce the mutual interference between sub-carriers, but also enhance the spectrum utilization. OFDM has the following advantages: (1) anti-multipath interference and narrowband interference than the single-carrier system strong; (2) use the system as compared to conventional frequency division multiplexing, OFDM system can maximize the use of spectrum resources; (3) make full use of the letter Bibi higher sub-channel noise, anti-frequency selective fading ability; (4) with time division, frequency division, code classification in various ways by combining the flexibility to support a variety of business.

Multiple-Input Multiple-Output Technology (MIMO). 4G system uses MIMO technology, which in a plurality of transmitting antennas and the receiving end, and GSM, CDMA IS - 95, 3G systems are not using this technique. Existing mobile communication systems, the majority of the base station antenna adopts a configuration out of two received. Comparison of these two technologies, MIMO system has the following advantages: (1) reducing the inter-symbol interference (ISI); (2) increase the spatial diversity gain; (3) improve the wireless channel capacity and spectral efficiency.

Smart Antenna Technique (SA). Smart Antenna is based on the principle of adaptive antenna mobile communication technology, it eliminate the interference, the expansion of the cell radius, reducing system cost and improve system capacity has unparalleled advantages. The technology uses multiple access (SDMA) technology space, the use of the difference in signal transmission direction, the same frequency or the same time slot, with the signal code channel distinguish dynamically change the coverage area of the signal, when the main beam is aligned direction of the user, or the side lobe aligned with the direction of interfering signals, and users can automatically track and monitor changes in the environment, provide excellent resources uplink and downlink signals for each user so as to suppress interference and accurately extract the effective signal purpose. This technology can not only improve the signal quality, increase transmission capacity can expand coverage and reduce system construction costs, so widely used in 4G systems.

Software Defined Radio (SDR) Technology. 4G mobile communication system, to achieve the desired communication mode "access to any person in any place, in any form of network", then at least to ensure that the mobile terminal is able to fit all types of air interface between different network environments can be no seam roaming, and can switch between different types of traffic. This means that the $4 \mathrm{G}$ system, the software will become very complicated. Therefore, the introduction of software-defined radio technology, the basic idea is to hardware as part of its universal basic platform to as many functions as wireless and personal communications by programmable software to achieve, making it a more work-band, multi-mode, multi-signal transmission and processing of the radio system. SDR core technology is wideband wireless receiver to replace the original narrow-band receivers and wideband analog / digital and digital / analog converter as close to the antenna, so that radio communication function can use as much as possible programming software. 


\section{Related Research on MIMO System}

MIMO-OFDM model and serial transmit bit stream through the circuit and the formation of many lines, but also form a bit stream, and the brightest to go through each of the bit stream coding, interleaving, and then carry out the corresponding mapping, and then the anti-protection inter channel interference intervals, and then performs OFDM modulation, delay spread to include such anti-prefix, then the results of the corresponding antenna out. Receiving end MIMO-OFDM system model is that when each receiving antenna receives a corresponding OFDM symbol and sync frequency of its setting, remove the corresponding CP, then its OFDM demodulates and then decodes, then it should channel estimation based on the results of the recovery and the received bit stream. To build a MIMO-OFDM system needs to achieve such synchronization, space-time processing, adaptive modulation and coding, channel estimation and other key technologies.

Frequency selective MIMO-OFDM channel is divided into a set of flat fading sub-channels at the same time also for the transmission of data, so you can easily expand the channel delay played a small effect. If the cyclic prefix length is longer than the maximum channel delay spread time, the receiver equalizer cannot be used for reception. Spectrum for each sub-carrier channels of this technology overlapping sub-channel of the frequency domain response of the peak will be able to point and other sub-channel of the frequency domain response of the combination of incense, so this only achieve the subcarrier orthogonality also allows spectrum resources are put to good use. Using FFT / IFFT can quickly and efficiently achieve the effect of modulation and demodulation. To take advantage of the channel characteristics can achieve adaptive power allocation for each carrier, so to choose modulation aspects of the use of conditions to improve system performance.

Compared with single carrier systems, this technique is very sensitive to the frequency deviation in the back. This may cause the transmitter or receiver local oscillator frequency to speed up, it will lead to the orthogonality of the subcarriers deviate, which would lead to the risk of the occurrence of the phenomenon. Peak to average power ratio of OFDM are high, when the phenomenon by the output signal from the OFDM signal is superimposed on a plurality of sub-channels is formed, if the phase of the signal is the same, the instantaneous power of the output signal will want more than the average power it has greatly improved the peak than the linear amplifier within the transmitter has a very high increase, said the added dynamic range of signal amplitude variations are not met, it is possible to make the signal waveform and spectrum distortion vary, so It may cause destruction of orthogonality of the subcarrier.

\section{G Mobile Communication Systems Link Scheme}

Uplink Data Encoding Scheme Design. Turbo code was chosen because it is the basis of innovation iterative decoding, maximum a posteriori probability decoding algorithm performed. The Turbo decoding method applied in block codes, you can establish a packet Turbo codes. Block Turbo code, the advantage is that he can be at a higher or lower SNR noisy environment can have anti-fading, anti-jamming, anti-obstruction performance. Block Turbo code when poor channel conditions for the application of the wireless communication system has a very good effect.

Block Turbo code can take advantage of using parity code and expand the Hamming code, a combination of both to reduce the difficulty of code decoding. Parity code is added to a parity bit at the end of each information packet; expand at the end of each Hamming code Hamming code word plus a dual efficacy bits. In the specific uplink and the downlink data encoding, mainly the following steps to implement:

BTC encoding is the need to transfer data communications, according to the order of information bits, efficacy bit matrix product was, in fact, a product code name is derived from this, it is noted that the two encoders inside and outside of the product code is not divided and it is a different order of the same set of information element is coded, which can encode press columns, then encode the resulting code word is identical. BTC encoded transmission line product codes may also be transmitted column transmission, it can also be transmitted in accordance with a diagonal manner. BTC code has very good flexibility in configuration, and it supports a wide range of bit rate decoder, 
so that even under very severe channel communication environment can also ensure that broadband bandwidth, bit error rate. At the same time, with a decoder can be repeated for a plurality of channels system variability, and each channel can be used by different rate. Coding block size can be predetermined specific encoding, modulation scheme, code rate, which can be achieved for the transmission of a predetermined block size coding, Turbo code re-use the packet compression. Shortening of BTC can be divided into two steps: The first step is coarse: the coded block being deleted row or column; next to fine-tune and fine tune to the first row of the individual bits of coded block removal, optional configuration application code and shorten, this will enable BTC code design more flexible and able to quickly match the election code.

BTC specific configuration code decoder for decoding is based on the test pattern formed by the channel information, to compile test pattern, the decoding process in the algebraic decoder is: choose $\mathrm{K}>>\mathrm{p}$ is an integer, where $\mathrm{k}$ information bits, $\mathrm{p}$ values 3,4,5, its bit value to compare, select the bit value of the position of the minimum reliability. , All of $2 \mathrm{p}$ associated bit sequence is substituted into the bit position of the minimum reliability of $p, 2 p$ obtain a test pattern. The resulting test patterns need to be passed to the hard decision decoder to compile and it can be drawn $2 p$ candidate code words. Finally, select and determine the final decode result in the candidate word mother.

The Down link Data Encoding Scheme Design. Downlink data transmission coding design, usually using cyclic shift matrix method to construct the $\mathrm{H}$ matrix can be generated QC-LDPC code having the quasi-cyclic characteristics, because this method is easier to implement in hardware and fast encoding algorithm rapid partial parallel decoding algorithm and acclaimed. Design downlink data coding scheme we chose to use more mature fast parallel decoding algorithm design.

Fast parallel decoding algorithm downlink data encoding scheme is a message passing algorithm, the transmission of information which each node is a probability way information is information or credible way. For example: check node $\mathrm{H}$ to pass information to the variable node $\mathrm{k}, \mathrm{H}$ is the information point to take some specific values can be expressed $\mathrm{H}$ point, then these specific value is defined as the probability information, which is the value corresponding to the $\mathrm{H}$ point observations and variable node connected with the $\mathrm{H}$-point pass on a message of confidence to the iteration check node $\mathrm{H}$, of course, does not include a variable node connected with the H-point $\mathrm{k}$. It may be the same probability information transmission of information to the contrary the variable node $\mathrm{k} \mathrm{H}$ checks nodes to transmit information.

\section{Conclusions}

With the development of $3 \mathrm{G}$ mobile communication technology, smart phones and tablet production, these intelligent terminal equipment has to meet the basic needs of users of the network, the application of 4G mobile communications technology will revolutionize the way people access the existing model, set visualization, multimedia and digital integration, transition Unicom relationship between people, between people and the network through high-speed data transmission efficiency will really into high-speed wireless Internet. Now transmitter and receiver can be set to transmit and receive multiple vice antennas are used, the use of MIMO technology enables the reliability of the channel and the channel capacity is improved, so that the error rate is reduced.

\section{References}

[1] Huifang Zhou: Information Technology, Vol. 6 (2004) No 53, p.25-26

[2] Hongli Zhang: Mobile Communications, Vol. 12 (2005) No 27, p.74-76

[3] Qin Guo: Telecom Engineering and Standardization, Vol. 1 (2006) No 33, p.11-14

[4] Jieming Liu: Electronic World, Vol. 3 (2007) No33, p.121-124 